Check for updates

Cite this: RSC Adv., 2017, 7, 42579

Received 10th July 2017

Accepted 24th August 2017

DOI: $10.1039 / \mathrm{c} 7 \mathrm{ra07601k}$

rsc.li/rsc-advances

\section{Comparison of morphological and functional restoration between asymmetric bilayer chitosan and bladder acellular matrix graft for bladder augmentation in a rat model $\dagger$}

\author{
Dongdong Xiao, (D) $\dot{t}^{\mathrm{a}}$ Qiong Wang, $\dot{+}^{\mathrm{b}}$ Hao Yan, ${ }^{\mathrm{a}}$ An Qin, ${ }^{\mathrm{c}}$ Xiangguo Lv, ${ }^{\mathrm{a}}$ Yang Zhao, \\ Ming Zhang, ${ }^{a}$ Zhe Zhou, ${ }^{a}$ Jiping $\mathrm{Xu}^{,}{ }^{\mathrm{d}}$ Qiaoling $\mathrm{Hu}^{\star \mathrm{e}}$ and Mujun Lu (D) *a
}

Because of the limitations of current scaffolds and unfavorable results of clinical trials, proper scaffolds facilitating bladder reconstruction are highly desirable. The aim of this study was to evaluate a novel asymmetric bilayer chitosan scaffold compared with conventional bladder acellular matrix graft (BAMG) in a rat model of bladder augmentation. Twenty-four 8 week-old male Sprague-Dawley rats were randomly assigned to the chitosan scaffold, BAMG and cystotomy groups. The rats' bladders were sampled for cystography and routine histological examination at 21 and 70 days. Immunofluorescence photometry, conscious cystometry, quantitative real-time polymerase chain reaction, and western blot analyses were performed using bladders at 70 days. Compared with BAMG, the chitosan scaffold consisted of a membrane-like compact layer and a sponge-like porous layer with an excellent combination of mechanical strength and flexibility. The chitosan group showed better performances than the BAMG group in radiographic cystography, smooth muscle regeneration, blood vessel numbers and functional restoration. In contrast to reduced bladder compliance induced by BAMG, bladder augmented by chitosan displayed nearly 1.5 -fold increased bladder capacity with comparable compliance to that of the cystotomy group at 70 days. The chitosan group exhibited higher levels of VEGF and VEGFR2, associated with the activation of the hypoxia-related SDF-1 $\alpha / C X C R 4$ pathway. These results suggested that the asymmetric bilayer chitosan scaffold is a promising scaffold for bladder reconstruction.

\section{Introduction}

Bladder augmentation is a surgery indicated in patients without adequate bladder capacity or detrusor compliance due to neuropathic causes (e.g., spinal cord injury, multiple sclerosis, myelodysplasia) or non-neuropathic causes (chronic,

\footnotetext{
${ }^{a}$ Department of Urology and Andrology, Ren Ji Hospital, School of Medicine, Shanghai Jiao Tong University, No. 145 Middle Shandong Road, Shanghai 200001, China. E-mail: lumujun@163.com; Fax:+86-21-63087768; Tel: +86-130-20156427

${ }^{b}$ Department of Urology, The Sun Yat-sen Memorial Hospital, Sun Yat-sen University, Guangzhou 510120, China

'Department of Orthopedics, Shanghai Key Laboratory of Orthopedic Implants, Shanghai Ninth People's Hospital, School of Medicine, Shanghai Jiao Tong University, Shanghai 200011, China

${ }^{d}$ Department of Urology, Shanghai Ninth People's Hospital, School of Medicine, Shanghai Jiao Tong University, Shanghai 200011, China

${ }^{e} \mathrm{MOE}$ Key Laboratory of Macromolecular Synthesis and Functionalization, Department of Polymer Science and Engineering, Zhejiang University, NO. 38 Zheda Road, Hangzhou 310027, China. E-mail: huql@zju.edu.cn; Tel: +86-139-06509096

$\dagger$ Electronic supplementary information (ESI) available. See DOI: $10.1039 / \mathrm{c} 7 \mathrm{ra0} 07601 \mathrm{k}$

¥ These authors contributed equally to this study.
}

interstitial, or radiation cystitis, detrusor instability, exstrophy, defunctionalized bladder). ${ }^{1-3}$ In 35 children's hospitals in the United States, 665 children with spina bifida underwent bladder augmentation over a 5 year period. ${ }^{4}$ Enterocystoplasty is the primary option for bladder augmentation, which is associated with a number of problems and complications, including malignancy, bowel obstruction, bladder perforation, urinary tract infection, and gastrointestinal dysfunction. ${ }^{5}$ In addition, when the intestine is used as tissue donor, some complications may arise such as improper bowel anastomosis, perforation, and calculi. ${ }^{6}$ Tissue donor complications could be avoided using tissue engineering. ${ }^{7}$

Various scaffolds have been tested to facilitate bladder tissue engineering, either supplemented with cells and/or growth factors. ${ }^{8}$ Unfortunately, postoperative bladder capacity was not augmented compared to preoperative status in large animals according to a systematic review. ${ }^{9}$ Although collagen-based scaffolds showed promising short-term outcomes in a pilot clinical trial, ${ }^{\mathbf{1 0}}$ the favorable outcomes in preclinical studies were not substantiated in recent clinical trials that tested the long-term follow-up of polyglycolic-acid (PGA)/poly 
(lactic-co-glycolic acid) (PLGA) copolymer and small intestinal submucosa (SIS) ${ }^{11,12}$ Synthetic polymers such as PGA and PLGA were denoted by its inclination to inflammation and fibrosis, as well as unsatisfied degradation rate. ${ }^{13}$ Naturally derived acellular biomatrices served as conventional scaffolds for tissueengineered bladder augmentation, represented by SIS, amniotic membrane and bladder acellular matrix graft (BAMG). BAMG has been extensively used with good biocompatibility and proved efficacy to support urothelial regeneration. ${ }^{14}$ Its failure in large bladder defect repair was largely owed to its insufficiency in facilitating smooth muscle regeneration, angiogenesis and innervation in our previous study. ${ }^{15}$ Moreover, scaffold shrinkage and perforation were frequently reported by simple application of BAMG or SIS. ${ }^{16,17}$

The current goals of scaffolds in bladder reconstruction are not only to support de novo tissue regeneration, but also to facilitate functional restoration. Showing high safety, good biocompatibility, diverse plasticity, and tunable biodegradability, chitosan is a proper candidate scaffold for bladder reconstruction. ${ }^{18}$ Approved by the US Food and Drug Administration for human use, chitosan is a natural copolymer derived from the alkaline deacetylation of chitin, which is derived from exoskeletons of crustaceans and from cell walls of fungi or insects. ${ }^{19}$ Its bacteriostatic and fungistatic properties are crucial for bladder reconstruction because the urinary tract is often colonized with various bacterial strains. ${ }^{20}$ Furthermore, the cationic nature of chitosan allows it to retain and concentrate a large number of cytokines and growth factors which are linked to anionic glycosaminoglycans, proteoglycans, and other negatively charged molecules. ${ }^{21}$

We hypothesized that our newly fabricated asymmetric bilayer chitosan scaffold could solve the aforementioned disadvantages of BAMG by increased porosity and strengthened mechanical properties. This study aimed to evaluate the novel asymmetric bilayer chitosan scaffold in a rat model of bladder augmentation compared with BAMG.

\section{Experimental}

\section{Animals}

Twenty-four 8 week-old male Sprague-Dawley rats matched for body weight were housed in wire-floored cages with free access to food and water in a temperature-controlled pathogen-free animal facility $\left(20-22{ }^{\circ} \mathrm{C}\right.$, humidity $40-70 \%, 12 / 12 \mathrm{~h}$ day per night cycle). The rats were acclimatized for a week before experiments. The rats were then randomized to the bilayer chitosan scaffold group (chitosan group), the BAMG group, and the cystotomy control groups (cystotomy group) ( $n=8$ /group). The rats in each group were further randomized to two evaluation time-points (21 and 70 days after operation) ( $n=4 /$ subgroup). Body weight was recorded before and after surgery. Post-operational bladder calculus was measured as number and total weight.

All animal procedures were approved and supervised by the Animal Experimental Ethical Inspection of Shanghai Ninth People's Hospital affiliated to Shanghai Jiao Tong University School of Medicine, under number HKDL[2016]149 and were performed in accordance with the guidelines of the China Act on Welfare and Management of Animals.

\section{Biomaterials}

The asymmetric bilayer chitosan scaffold was made using a novel self-deposition technique developed by our team. ${ }^{22}$ Briefly, chitosan (Haihui Bioengineering Co. Ltd., Qingdao, China) was dissolved in $1 \%(\mathrm{v} / \mathrm{v})$ acetic acid to generate a $2.5 \%$ (w/v) solution. The solution was precipitated with $1 \mathrm{M} \mathrm{NaOH}$ added dropwise to form micro-hydrogels. Then, these microhydrogels were washed to neutral $\mathrm{pH}$ with tri-distilled water and poured in the mould for self-deposition and to drain off the remaining water. The compact membrane-like layer was obtained by drying the micro-hydrogels at $60^{\circ} \mathrm{C}$ under vacuum, while the porous sponge-like layer was achieved by freezedrying. Using sprayed medical $\alpha$-cyanoacrylate, the two layers were combined (mass ratio of $3: 2$ ) at room temperature for 1 min to obtain the asymmetric bilayer chitosan scaffold.

BAMG was made according to the decellularization protocol used in our previous work, in which complete elimination of cellular nuclei was confirmed by histology and residual DNA. ${ }^{23}$ Briefly, porcine bladders were harvested from 3 month-old pigs (Shanghai Super-B\&K Laboratory Animal Co. Ltd., Shanghai, China). After separated from adjacent adipose tissue and facia, and they were rinsed in $4{ }^{\circ} \mathrm{C}$ phosphate-buffered saline (PBS; pH 7.2-7.4). Reserved from surgical delamination of urothelium, muscle and serosal layers, lamina propria was treated sequentially by distilled water in a stirring flask $\left(200 \mathrm{rpm}, 4^{\circ} \mathrm{C}, 48 \mathrm{~h}\right)$, $0.03 \%$ trypsin (Gibco, Thermo Fisher Scientific Inc., MA, USA) at $37{ }^{\circ} \mathrm{C}$ for $1 \mathrm{~h}, 0.2 \%$ Triton $\mathrm{X}-100$ and $0.1 \%$ ( $/ \mathrm{v} / \mathrm{v}$ ) ammonium hydroxide at $37^{\circ} \mathrm{C}$ for 7 days. The solution was refreshed every day. The resulting matrix was washed with distilled water at $4{ }^{\circ} \mathrm{C}$ for 2 days and stored in $75 \%$ ethanol. All chemicals were purchased from Sigma-Aldrich Corporation, NY, USA.

\section{Scanning electron microscopy (SEM)}

A SEM was used to observe scaffolds' surface morphology, inner space, and thickness as previously described. ${ }^{24}$ Chitosan and BAMG scaffolds were cut into $7 \times 7 \mathrm{~mm}$ pieces and prefixed with $2 \%$ glutaraldehyde at $4{ }^{\circ} \mathrm{C}$ for $2 \mathrm{~h}$, washed twice with PBS, and post-fixed in $1 \%$ osmic acid at $4{ }^{\circ} \mathrm{C}$ for $2 \mathrm{~h}$. After two washes with distilled water, the samples were dehydrated with gradient ethanol and dried to a critical point. The samples were then mounted, sputter-coated with gold in a caster (NeoCaster, MP1920NCTR, JEOL, Tokyo, Japan), and examined under a SEM (NeoScope, JCM-5100, JEOL, Tokyo, Japan) at cross-section, topview, and bottom-view at $20-25 \mathrm{kV}$ with different magnifications.

\section{Micro-computed tomography $(\mu \mathrm{CT})$}

The microstructure analysis of the bilayer chitosan was performed by the $\mu$ CT system (SkyScan-1176, Bruker microCT, Kontich, Belgium). Because the BAMG is a single-layer membrane with dense structure, it was not investigated by the $\mu$ CT system. Scans of the whole bilayer chitosan scaffold $(2.6 \times 3.0 \mathrm{~cm})$ were performed using $8.96 \mu \mathrm{m}$ voxel size, $40 \mathrm{kV}, 2000 \mu \mathrm{A}$ and 0.45 degrees rotation step. The compact layer of the chitosan scaffold was not evaluated 
because of its dense structure. For the porous layer of the chitosan scaffold, $\mu \mathrm{CT}$ evaluation was performed on four randomly selected cylinders regions with a diameter of $0.3 \mathrm{~mm}$ and height of $7.0 \mathrm{~mm}$. After three-dimensional reconstruction, the CT Analyzer 1.16.4.1 (Bruker microCT, Kontich, Belgium) was used for microstructure analysis. The indexes including pore size, porosity and pore interconnectivity were calculated for the porous layer of the chitosan scaffold.

\section{Mechanical properties}

Each scaffold was cut into a dog-bone shape $(10 \times 40 \mathrm{~mm})$ and hydrated in PBS for 1 day to reach a swelling equilibrium. Samples were subjected to mechanical testing using a biomechanical analyzer (Instron 5542, Illinois Tool Works Inc., IL, USA) to measure maximal load and elastic modulus as previously described..$^{25}$ The interval length of the two grippers was set at $10 \mathrm{~mm}$ with a gradual moving speed of $25 \mathrm{~mm} \min ^{-1}$ until the complete rupture of the scaffolds.

\section{Bladder augmentation, catheterization, and conscious cystometry}

After anesthesia by intraperitoneal injection of pentobarbital (30 $\mathrm{mg} \mathrm{kg}^{-1}$ ), the bladder was exposed (Fig. 1A) and fixed by atraumatic forceps (Fig. 1B). The anterior portion of the bladder that was immediately distal to the dome was incised longitudinally in the midline (approximately $1 \mathrm{~cm}$ ) (Fig. 1C). The bladder defect was marked by four non-absorbable 5-0 polypropylene sutures in each corner, and anastomosed with scaffolds by absorbable 8-0 polyglactin sutures (Ethicon, Johnson \& Johnson Services, Inc., NJ, USA) in a running continuous fashion (Fig. 1D). The cystotomy group underwent bladder incision and immediate closure with the same incision size.

Prior to conscious cystometry at 70 days after operation, the bladder was catheterized with a PE-50 tube as previously described. ${ }^{26}$ A slack purse-string suture was made on the dome of the bladder, in the center of which a hole opening was pierced by an $18 \mathrm{G}$ needle (Fig. 1E). The PE-50 tube was inserted through the opening and the purse-string suture was pulled tight (Fig. 1F). Room temperature normal saline was infused into the bladder to test the suture (Fig. 1G). The dorsal PE-50 tube was coiled in a subcutaneous pouch in the neck (Fig. $1 \mathrm{H}$ ), which was closed with interrupted 5-0 polypropylene sutures. To prevent infections, $30 \mathrm{mg} \mathrm{kg}^{-1}$ cefazolin sodium was administered subcutaneously.

On the day of conscious cystometry, the coiled PE-50 tube was released from the subcutaneous pouch and connected to a pressure transducer (Laborie Medical Technologies, Brossard, Canada) and an infusion pump (infusion speed: $35 \mathrm{ml} \mathrm{h}^{-1}$ ) via a 3-way stopcock. The rat was placed in a metabolic cage without restraint. Its voiding volume was recorded by a gravity transducer. After the intra-vesical pressure curve became stable, at least three micturition cycles were recorded for urodynamic parameters analysis. Cystometrogram was drawn using the Origin 9.0 (Origin Lab, Northampton, MA, USA).

\section{Retrograde cystography and gross bladder morphology}

At 21 and 70 days post implantation, the rats' bladders were injected with contrast medium (30\% iopamidol, GE Healthcare, IL, USA) through intra-vesical instillation until first urethral urine leakage under general anesthesia (pentobarbital, $30 \mathrm{mg}$ $\mathrm{kg}^{-1}$, intraperitoneal). X-ray film was obtained for each experimental subject. Afterwards, the bladder was extruded and the gross morphology was examined in vivo.

\section{Bladder calculus analysis}

The bladder calculus compositions were analyzed by an infrared spectrum automatic analysis instrument (LIIR-20, Lanmode Scientific Instrument Co. Ltd., Tianjin, China) according to
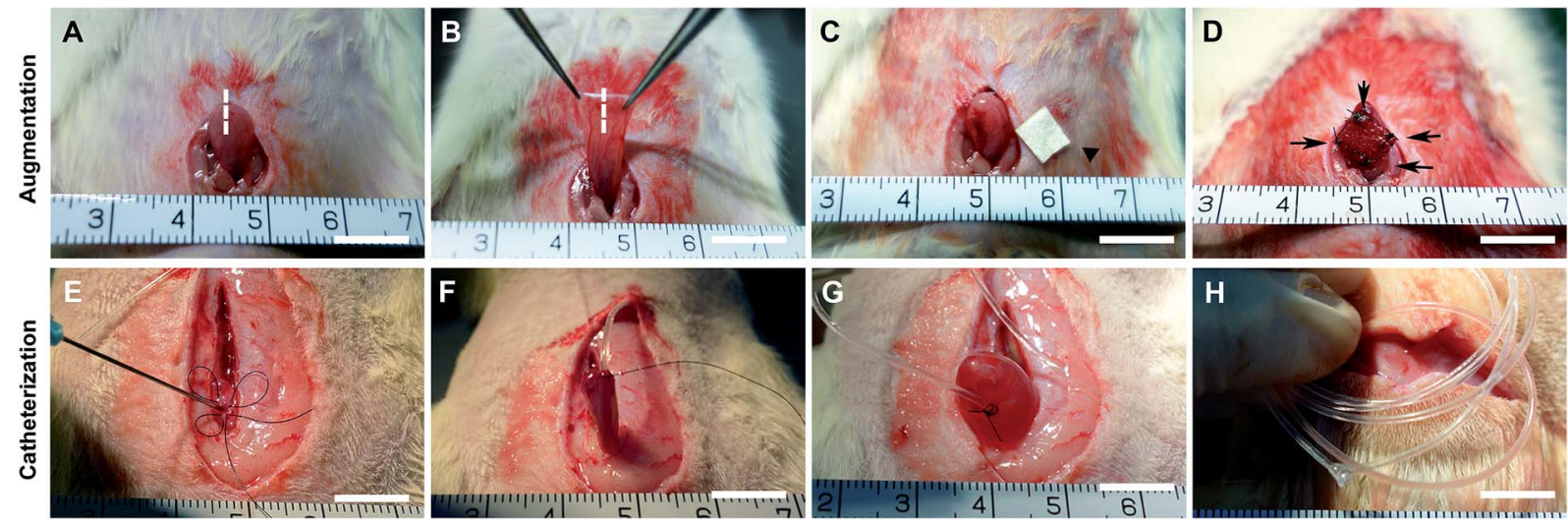

Fig. 1 Surgical procedures of bladder augmentation with the chitosan scaffold and bladder catheterization with a PE-50 tube. (A) The abdomen was dissected and the bladder was extruded outside the peritoneal cavity. (B) The bladder was fixed by forceps beside the apex. (C) A longitudinal incision of approximately $1 \mathrm{~cm}$ was made in the bladder midline. (D) The chitosan scaffold $(7 \times 7 \mathrm{~mm})$ was anastomosed to the bladder defect. (E) A purse-string suture was made at the dome of the bladder, in the center of which a hole was made using an $18 \mathrm{G}$ needle. (F) A PE-50 tube was inserted into the opening and the purse-string suture was pulled tight. (G) The suture seal was tested by normal saline infusion. (H) The dorsal PE50 tube was coiled in a subcutaneous pouch. Scale bar $=1 \mathrm{~cm}$. Dashed lines mark the incision area. Black triangles indicate the chitosan scaffold. Black arrows point out the marking sutures between the native bladder and the chitosan scaffold. 
manufactural protocol. Bladder calculus was rinsed with distilled water and then dried at room temperature. The samples were rubbed into powder, mixed with potassium bromide $(\mathrm{KBr})$ at a ratio of $1: 50$, and pressed into thin slices (thickness of $1 \mathrm{~mm}$ ) under spectrum analysis.

\section{Histology}

The rats were euthanized by $\mathrm{CO}_{2}$ asphyxiation and the bladders were excised for standard histological processing at 21 and 70 days post implantation. ${ }^{27}$ After embedding in paraffin in an axial orientation to capture the entire circumferential surface, bladder sections $(10 \mu \mathrm{m})$ were cut and stained with hematoxylin and eosin (H\&E) and Masson's trichrome stains (MTS). Immunofluorescence analysis was carried out to detect cytokeratin (CK, AE1/AE3) for urothelium, $\alpha$-smooth muscle actin ( $\alpha$-SMA) for smooth contractile muscle, CD31 for vessels, and NeuN for neuron buttons at 70 days post-operation. The following primary antibodies were used: anti-cytokeratin AE1/AE3 (1 : 50 dilution, ab1747070, Abcam, Cambridge, MA, USA), anti- $\alpha$-SMA ( 1 : 150 dilution, ab32575, Abcam, Cambridge, MA, USA), antiCD31 (1:100 dilution, ab119339, Abcam, Cambridge, MA, USA), and anti-NeuN (1 : 150 dilution, ab177487, Abcam, Cambridge, MA, USA). Sections were incubated with speciesmatched fluorescein isothiocyanate (FITC)-conjugated secondary antibodies (Millipore, Billerica, MA, USA) and nuclei were counterstained with 4',6-diamidino-2-phenyllindole (DAPI). Specimens were visualized using a Nikon Eclipse 80i fluorescence microscope (Nikon Instruments Inc., Tokyo, Japan) and representative images were acquired using NISElements 4.0 (Nikon Instruments Inc., Tokyo, Japan). The percentages of the corresponding protein positive expression per total area were calculated by ImageJ $1.50 \mathrm{i}$ (National Institutes of Health, Bethesda, MD, USA) from six randomly selected fields of three slides.

\section{Quantitative real-time polymerase chain reaction (qRT-PCR)}

Total RNA was extracted from the regenerated bladder tissue using Trizol (Invitrogen Inc., Carlsbad, CA, USA). cDNA was synthesized by reverse transcription of total RNA using the Hiscript Reverse Transcriptase (Vazyme Biotech Co. Ltd., Nanjing, China), according to the manufacturer's instructions. cDNA products were diluted 10 times, $4 \mu \mathrm{l}$ was used as the templates for qRT-PCR. The expression of vascular endothelial growth factor (VEGF), VEGF receptor 2 (VEGFR2), stromal cellderived factor-1 $\alpha$ (SDF-1 $\alpha)$, and CXC chemokine receptor 4 (CXCR4) was determined in an Eco $^{\text {TM }}$ Real-Time PCR System (ABI7900, Illumina, Inc., San Diego, CA, USA) with the Power SYBR Green PCR master mix $(2 \times)$ (Vazyme Biotech Co. Ltd., Nanjing, China). qRT-PCR was performed with a protocol of: (1) $50{ }^{\circ} \mathrm{C}$ for $2 \mathrm{~min}$; (2) $95^{\circ} \mathrm{C}$ for $10 \mathrm{~min}$; and (3) 40 cycles at $95^{\circ} \mathrm{C}$ for $30 \mathrm{~s}$ and $60{ }^{\circ} \mathrm{C}$ for $30 \mathrm{~s}$. The mRNA relative expression level of each gene was normalized to $\beta$-actin by $2^{-\left(\Delta \Delta C_{t}\right)}$. The primers for qRT-PCR are listed in Table 1 . Three bladders from each group at 12 weeks post-operation were used for qRT-PCR. Each assay was performed in triplicate.

\section{Western blot analysis}

The protein in the bladder samples were harvested by radioimmunoprecipitation assay (RIPA) lysis buffer, quantified by a bicinchoninic acid (BCA) Protein Assay Kit (Beyotime Biotechnology, Shanghai, China), and separated by $10 \%$ sodium dodecyl sulphate polyacrylamide gel electrophoresis. Then, the samples were subjected to immunoblotting with antibodies to VEGF (1: 1000, ab32152, Abcam, Cambridge, MA, USA), VEGFR2 (1 : 300, Sc-6251, Santa Cruz Biotechnology, Inc., Dallas, TX, USA), SDF-1 $\alpha$ (1:500, ENT4225, Elabscience Biotechnology Co. Ltd., Wuhan, China), and CXCR4 (1 : 100, ab124824, Abcam, Cambridge, MA, USA), and were then transferred onto polyvinylidene fluoride membranes (Millipore, Darmstadt, Germany). The membranes were blocked in 5\% non-fat milk for $2 \mathrm{~h}$ at room temperature and incubated with primary antibodies overnight at $4{ }^{\circ} \mathrm{C}$, followed by incubation with horseradish peroxidase-conjugated secondary antibodies (BA1054, 1 : 50 000, Boster Biological Technology Co. Ltd., Wuhan, China) for $2 \mathrm{~h}$ at room temperature. The protein bands were visualized with an enhanced chemiluminescence detection kit (NCI5079, Thermo Fisher Scientific Inc., MA, USA) on $\mathrm{X}$-ray films (XBT-1, Eastman Kodak Company, NY, USA). $\beta$-Actin (1:200, BM0627, Boster Biological Technology Co. Ltd., Wuhan, China) was used as an internal control.

\section{Statistical analysis}

All data were expressed as mean \pm standard deviation. Statistical analyses were performed using two-tailed Student's $t$-test or one-way analysis of variance (ANOVA) with the Bonferroni post hoc test in GraphPad Prism 5.01 (GraphPad Software Inc., San Diego, CA, USA). Two-sided $P$-values $<0.05$ were considered statistically significant.

\section{Results and discussion}

\section{Bilayer configuration and mechanical properties of the chitosan scaffold}

On the contrary to the approximately $100 \mu \mathrm{m}$ thick and relatively dense structure of BAMG with a rough side and a smooth side, the chitosan scaffold presented a tightly interfaced bilayer configuration of approximately $600 \mu \mathrm{m}$ thickness (Fig. 2A, ESI Fig. $1 \dagger$ ). The asymmetric bilayer chitosan scaffold consisted of a membrane-like compact layer serving as the water barrier towards the bladder cavity, and a sponge-like porous layer for regenerated tissues ingrowth towards the peritoneal cavity (ESI Fig. $2 \dagger)$. The porous layer of chitosan had pores on its surfaces and internal space, ensuring plenty of space for the ingrowth of regenerative cells. The pore size is $0.1205 \pm 0.005900 \mathrm{~mm}$, the porosity is $93.02 \pm 0.4470 \%$, the pore interconnectivity is $(1876 \pm 257.6) / \mathrm{mm}^{3}$. Meanwhile, the compact membrane possessed a dense texture with no apparent pore even at high magnification. Although the high porosity reduced the mechanical strength of the porous layer, the compact layer endowed the entire bilayer chitosan scaffold with stronger mechanical properties than BAMG. Despite its similar maximum load with BANG $(9.79 \pm 3.01$ vs. $5.65 \pm 2.73 \mathrm{~N}, P=0.15)$ (Fig. 2B), 
Table 1 Oligonucleotide sequence for qRT-RCR primers

\begin{tabular}{|c|c|c|}
\hline mRNA & Sense strand $\left(5^{\prime}-3^{\prime}\right)$ & Antisense strand $\left(5^{\prime}-3^{\prime}\right)$ \\
\hline SDF-1 $\alpha$ & ATGCCCCTGCCGATTCTTTG & TTGTTGCTTTTCAGCCTTGC \\
\hline CXCR4 & CGGTCATCCTTATCCTGGCT & CTCTTGAATTTGGCCCCGAG \\
\hline VEGFR2 & CTTCATAATAGAAGGCGTCCAG & ATAAGGCAAGCGTTCACAGC \\
\hline$\beta$-Actin & CACGATGGAGGGGCCGGACTCATC & TAAAGACCTCTATGCCAACACAGT \\
\hline
\end{tabular}

the chitosan scaffold performed significantly better in elastic modulus than BAMG $(18.61 \pm 0.48 v s .12 .95 \pm 2.34 \mathrm{MPa}$, $P=0.01$ ) (Fig. 2C). These results indicated that the chitosan scaffold had higher porosity and better mechanical properties than BAMG.

\section{Characteristics of the animal models}

All animals survived for the experimental period. Both scaffolds groups gained similar body weight with the control group. No bladder calculus was observed in the cystotomy group. The chitosan and BAMG groups had comparable bladder calculi (Table 2), whose majority composition was ammonium magnesium phosphate. These results suggested that the chitosan scaffold did not affect the metabolism of the animals drastically.

The safety of scaffolds is of prior concern for clinical application, as well as the biodegradability in the regeneration process. Unlike the acidic remnants produced by degrading synthetic polymers of PGA/PLGA, the bilayer chitosan scaffold is metabolized into non-toxic D-glucosamines by lysozymes and tuned with suitable degradation speed for bladder repair, which alleviates the systematic toxicity and the local inflammation reaction, and eliminates the risk of nidus for large bladder calculus. $^{28}$ Although a small quantity of bladder calculus was induced by the chitosan scaffold, it facilitated bladder regeneration with trivial scaffold shrinkage, fibrosis and metabolic disturbance.

\section{Morphological restoration in gross appearance and retrograde cystography}

At 21 days, both scaffolds entirely supported the original implantation areas with trivial fibrosis and contracture, which presented as small depressions in the bladder wall on retrograde cystography (Fig. 3). They were finally replaced by de novo host bladder tissue with remaining marking sutures at 70 days. The regenerated bladder walls in the chitosan group recovered to smooth oval shape, but the original defect site in the BAMG group still presented an irregular shape with depression under cystography. There was no leakage of contrast medium, fistula, or diverticulum in all rats. These results suggested that the
A

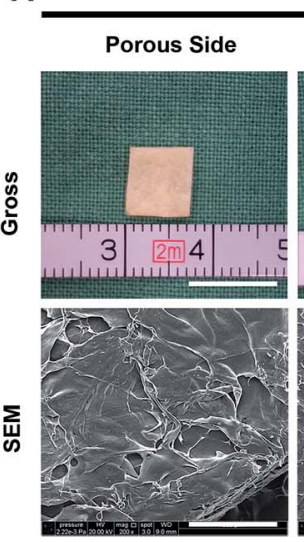

B

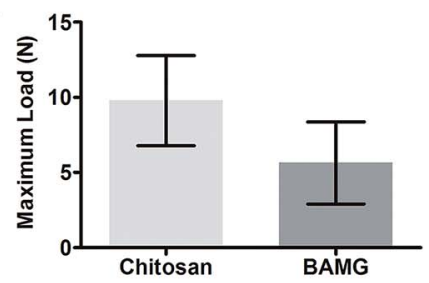

Chitosan
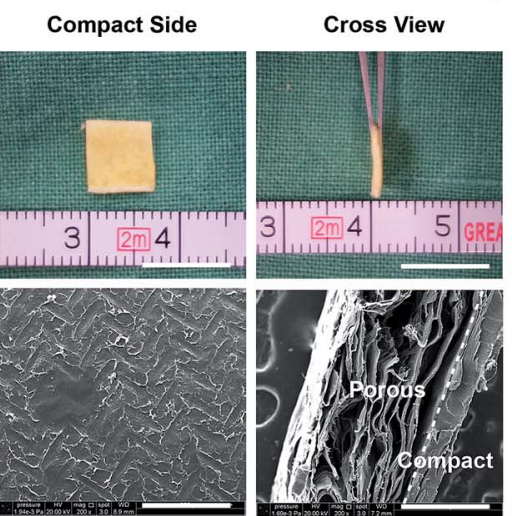

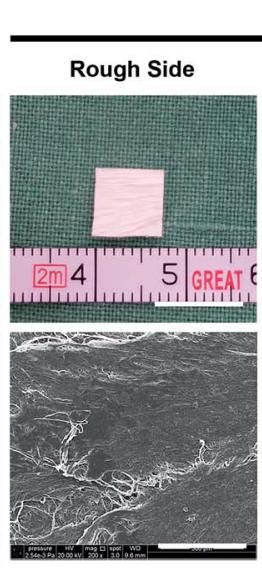

BAMG
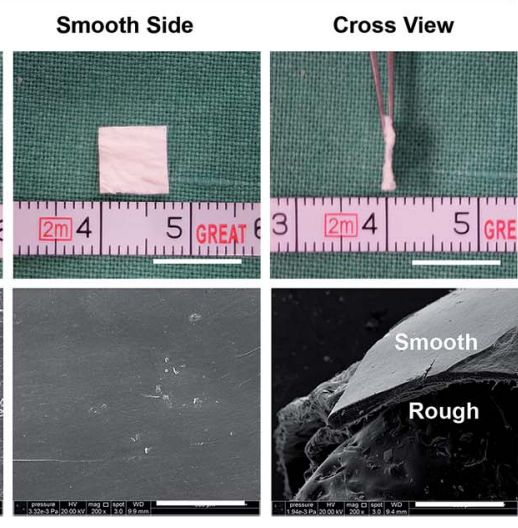

C

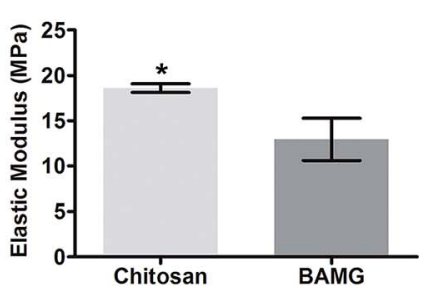

Fig. 2 Structural and mechanical comparisons between the chitosan scaffold and BAMG. (A) Gross view and representative SEM photomicrographs displaying the structure of the scaffolds. The dash line distinguishes the porous and compact layers of the chitosan scaffold. Gross view scale bar $=1 \mathrm{~cm}$. SEM scale bar $=500 \mu \mathrm{m}$; (B) comparison of maximal load and (C) elastic modulus of the chitosan scaffold and BAMG. $* P<$ 0.05 vs. the BAMG group. All experiments were performed in triplicate. 
Table 2 Body weight change and bladder calculus at 21 and 70 days after bladder augmentation

\begin{tabular}{|c|c|c|c|c|c|}
\hline & Weight pre-op (g) & Weight post-op (g) & Weight change (g) & Stone number $(n)$ & Stone weight $(\mu g)$ \\
\hline \multicolumn{6}{|l|}{21 days } \\
\hline Chitosan & $209.7 \pm 4.5$ & $336.1 \pm 8.7$ & $126.4 \pm 7.7$ & $1.5 \pm 0.5$ & $3.0 \pm 1.0$ \\
\hline BAMG & $202.2 \pm 9.4$ & $329.3 \pm 9.8$ & $127.1 \pm 15.8$ & $2.2 \pm 0.8$ & $4.5 \pm 1.5$ \\
\hline Cystotomy & $205.9 \pm 7.4$ & $331.5 \pm 9.5$ & $125.7 \pm 14.1$ & 0 & 0 \\
\hline \multicolumn{6}{|l|}{70 days } \\
\hline Chitosan & $207.9 \pm 8.8$ & $381.2 \pm 8.4$ & $173.3 \pm 8.8$ & $3.5 \pm 1.4$ & $6.9 \pm 2.5$ \\
\hline BAMG & $207.5 \pm 8.6$ & $380.9 \pm 8.1$ & $173.4 \pm 12.6$ & $4.2 \pm 1.8$ & $8.2 \pm 3.4$ \\
\hline Cystotomy & $206.2 \pm 8.8$ & $383.6 \pm 8.9$ & $177.4 \pm 14.4$ & 0 & 0 \\
\hline
\end{tabular}
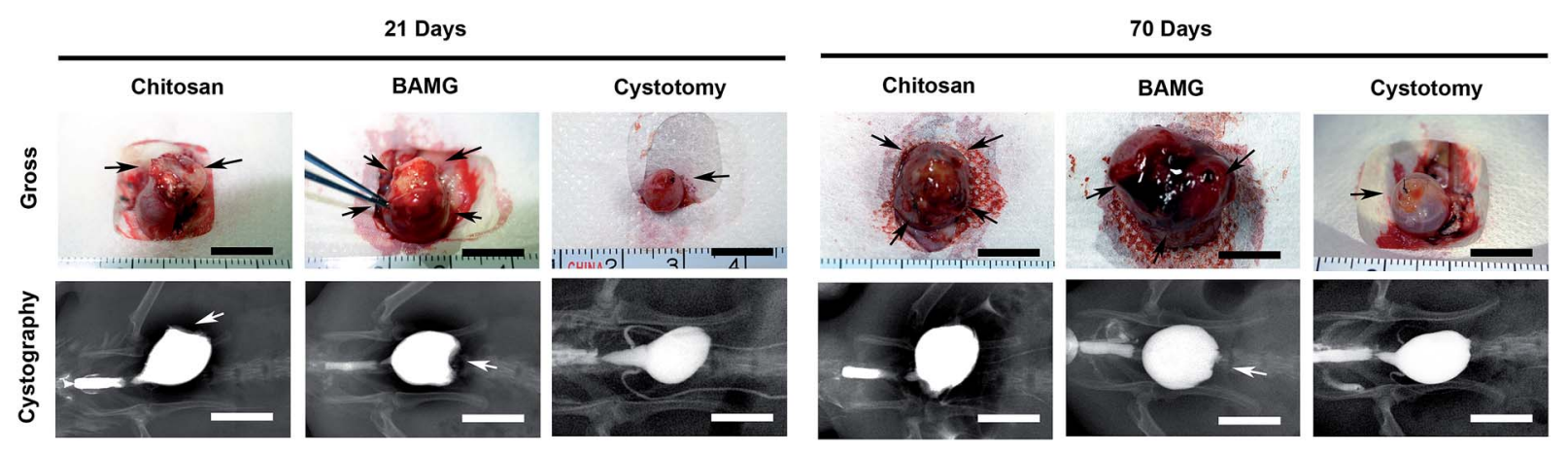

Fig. 3 Gross morphology of regenerated tissue and retrograde cystography at 21 and 70 days after bladder augmentation. Black arrows mark the remaining suture and regenerated tissue present in vivo within the original implantation sites supported by scaffolds. White arrows point out depression of regenerated area under retrograde cystography. Scale bar $=1 \mathrm{~cm}$.

chitosan scaffold could lead to better morphological regeneration of the bladder wall after bladder augmentation.

\section{Improved bladder smooth muscle regeneration and vessel numbers by the chitosan scaffold}

When focusing on the regenerated area, both scaffolds were lined with multilayer urothelium bordered by lamina propria at 21 and 70 days. Sparse smooth muscle bundles concentrated at the borders of the regeneration areas at 21 days, and traversed the entire width of the implants at 70 days after surgery (Fig. 4).

In addition, histological comparison among groups were analyzed by immunofluorescence (Fig. 5A). There were more CK-positive areas in the chitosan and BAMG groups compared with the cystotomy group at 70 days $(P<0.01)$ (Fig. 5B). The urothelial layer in the chitosan and BAMG groups presented irregular hyperplasia (Fig. 5A). The chitosan group showed more $\alpha$-SMA-positive smooth muscle cells than the BAMG $(P<$ $0.01)$, which was close to that of cystotomy groups $(P>0.05)$ (Fig. 5C). The same tendency was also observed with respect to CD31-positive vessel numbers. The mean number of CD31positive vessels was similar in the chitosan and cystotomy groups $(P>0.05)$, which were significantly higher than that of the BAMG group $(P<0.01)$ (Fig. 5E). However, CD31-positive vessel diameter was comparable in the chitosan and BAMG groups, but was significantly lower in the chitosan group compared with the cystotomy group $(P<0.01)$ (Fig. 5F). Both chitosan and BAMG groups presented lower rates of NeuN- positive cells than that of the cystotomy group $(P<0.01)$ (Fig. 5D). These histology results suggested that the chitosan scaffold could lead to proper bladder wall regeneration, expect for vessel diameters and innervation.

Simple application of BAMG had been reported to facilitate multi-layer urothelium regeneration in $60-70 \%$ partial cystectomy, ${ }^{17}$ which demonstrated the high regenerated potency of urothelium. There was abundant regenerated urothelium in both chitosan and BAMG groups at 21 days, and developed to multi-layer structure with hyperplasia at 70 days. The urothelial hyperplasia was also found in regenerated bladder tissue supported by BAMG-silk fibroin at 12 weeks in our previous study, which was associated with chronic local inflammation of macrophages. ${ }^{23}$ This conclusion was verified by the majority composition of bladder calculus, ammonium magnesium phosphate, which was recognized to be caused by the inflammatory response. ${ }^{29}$ Anti-inflammation approaches were desirable under comprehensive examination.

The insufficient smooth muscle regeneration, angiogenesis and resultant impeded function were major problems of the biological scaffolds lack adequate porosity. In its first published clinical trial for bladder augmentation, SIS repaired 5 patients suffering from bladder extrophy with disappointing histological results of smooth muscle, which was confirmed by the functional tests with a low increase of bladder capacity and compliance at 6 and 18 months respectively. ${ }^{12}$ Although the bladder capacity and compliance were greatly improved by SIS in patients with myelomeningocele or spinal cord injury in 


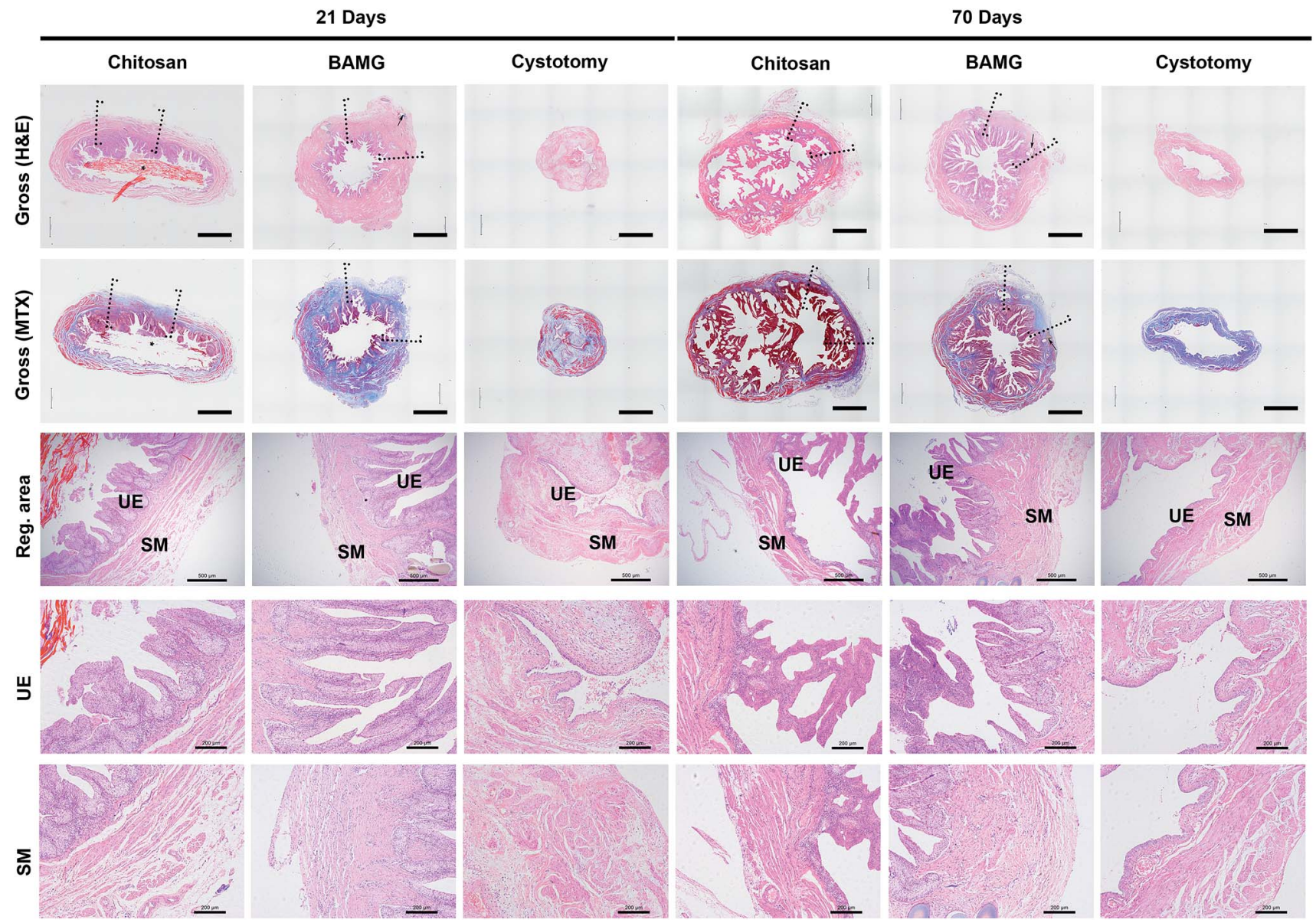

Fig. 4 Histological comparisons among the chitosan, BAMG, and cystotomy groups at 21 and 70 days after operation. Representative photomicrographs of entire bladder transverse sections by H\&E and MTS stains $(40 \times)$, bladder regenerated areas (Reg. area, $100 \times$ ), magnified de novo urothelium (UE, 200×), and smooth muscle (SM, 200×) areas by H\&E stain. Reconstructed areas are labelled by black brackets. (*) marks the chitosan scaffold. Whole scan: $40 \times$, scale bar $=3$ mm; Reg. area: $100 \times$, scale bar $=500 \mu \mathrm{m}$; UE and SM: $200 \times$, scale bar $=200 \mu \mathrm{m}$.

a recent study, smooth muscle was unfavorably regenerated in small amounts. ${ }^{30}$ The favorable regeneration of $\alpha$-SMA-positive smooth muscle bundles in the chitosan group was partially credited to its increased porosity. In addition, the good biocompatibility of chitosan with smooth muscle cells should be noted for this phenomenon. ${ }^{31}$ Three mechanisms may explain smooth muscle regeneration in the chitosan scaffold: (a) mature cells migration in the neighborhood; (b) dedifferentiation of mature cells prior to migration and redifferentiation; and (c) myofibroblasts infiltration followed by differentiation into smooth muscle cells. ${ }^{32}$ The clinical impotency to facilitate smooth muscle regeneration of SIS was consolidated by its counterpart BAMG in our previous study in animal models, which was associated with the paucity in angiogenesis. ${ }^{15}$

Improper nerve regeneration would impede bladder functional restoration on the long term. ${ }^{33}$ Although chitosan was shown to promote neural cells attachment and differentiation, ${ }^{34}$ the re-innervation rate still could not recover to normal levels by the bilayer chitosan scaffold after 70 days. It was reported that chitosan fibers supported the adhesion, migration, and proliferation of Schwann cells, which allowed for axonal regeneration in the peripheral nervous system. ${ }^{35}$ In vivo studies also suggested that chitosan-based membranes showed promising results regarding its applications in peripheral nerve engineering. ${ }^{36}$ One possible explanation of the impeded innervation in chitosan group is that the process of nerve regeneration could take as long as 20 weeks. ${ }^{37}$ As a result, a longer repair time is required unless the nerve regeneration would be accelerated.

\section{Augmented bladder capacity with physiological compliance after bladder augmentation by chitosan scaffold}

All three groups exhibited regular filling and voiding phases with the normal micturition patterns without signs of hypercontraction, obstruction, or irritation (Fig. 6A). Threshold pressures were comparable between the chitosan and cystotomy groups, while the BAMG group showed significant gain in threshold pressure compared to the cystotomy group $(P<0.05)$. Basal and peak pressures were comparable among the three groups (Fig. 6B). Voiding volume and bladder capacity were significantly enlarged to nearly 1.5 -fold after bladder augmentation with the chitosan scaffold and BAMG compared to the cystotomy group $(P<0.01$ for voiding volume, $P<0.001$ for 

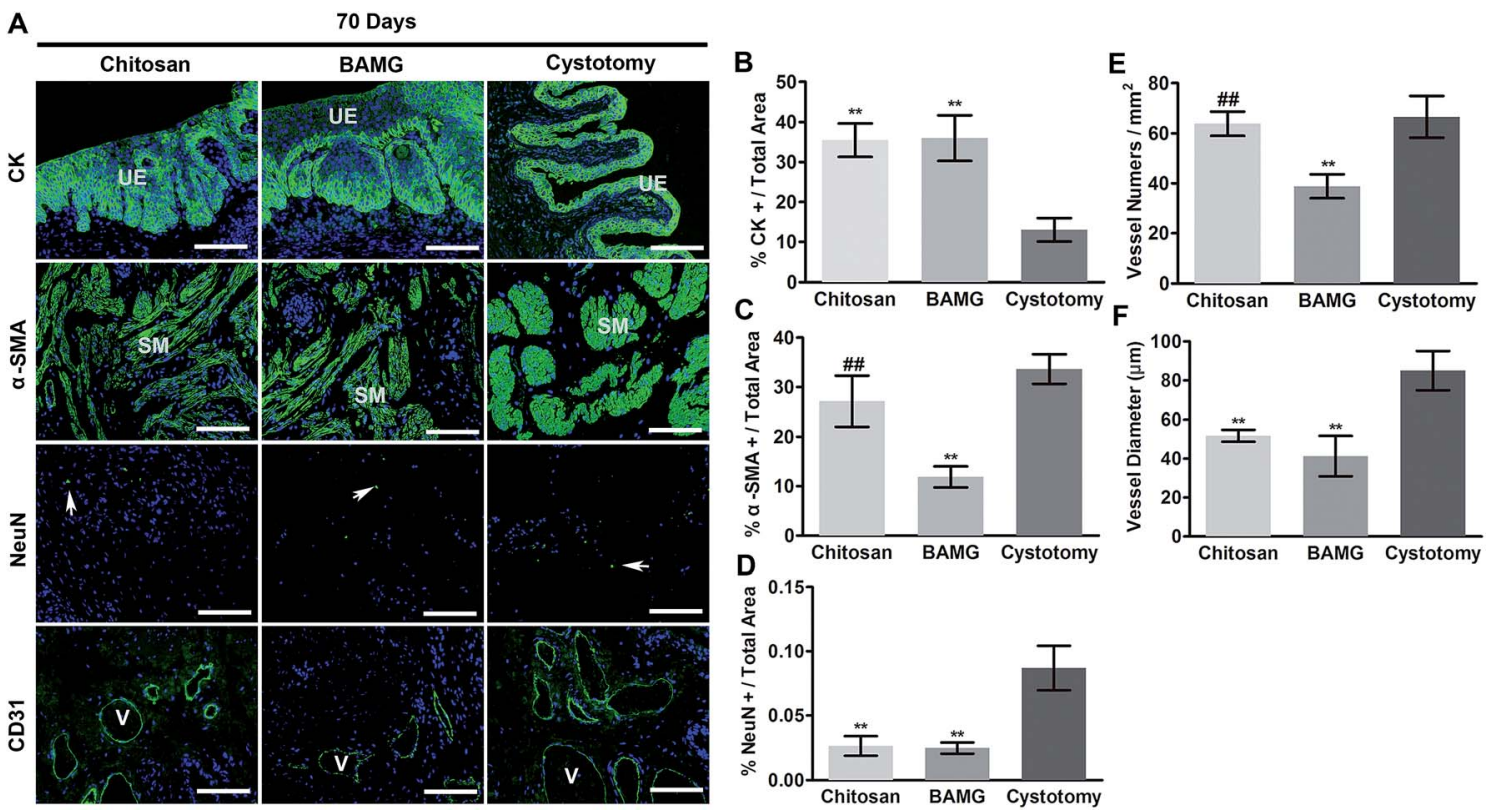

Fig. 5 Immunofluorescence and histomorphometric comparison of the regenerated bladders among the chitosan, BAMG, and cystotomy groups at 70 days after operation. (A) Representative immunofluorescence of urothelium (UE) marker CK, smooth contractile muscle (SM) marker $\alpha$-SMA, neuronal marker (NeuN, showed by white arrows), and blood vessels (showed by " $V$ ") endothelial marker (CD31). Positive respective marker expression is shown in green (labelled by FITC) and the nuclei are counterstained in blue by DAPI (blue). $200 \times$, scale bar $=100 \mu \mathrm{m}$. Histomorphometric quantitative comparison of (B) CK-positive urothelium; (C) $\alpha$-SMA-positive smooth muscle bundles; (D) NeuN-positive neuronal boutons; and (E) mean number per $\mathrm{mm}^{2}$ and (F) mean diameter of CD31-positive vessels. ${ }^{* *} P<0.01 \mathrm{vs}$. the control group; ${ }^{\# \#} P<0.01 \mathrm{vs}$. the BAMG group.
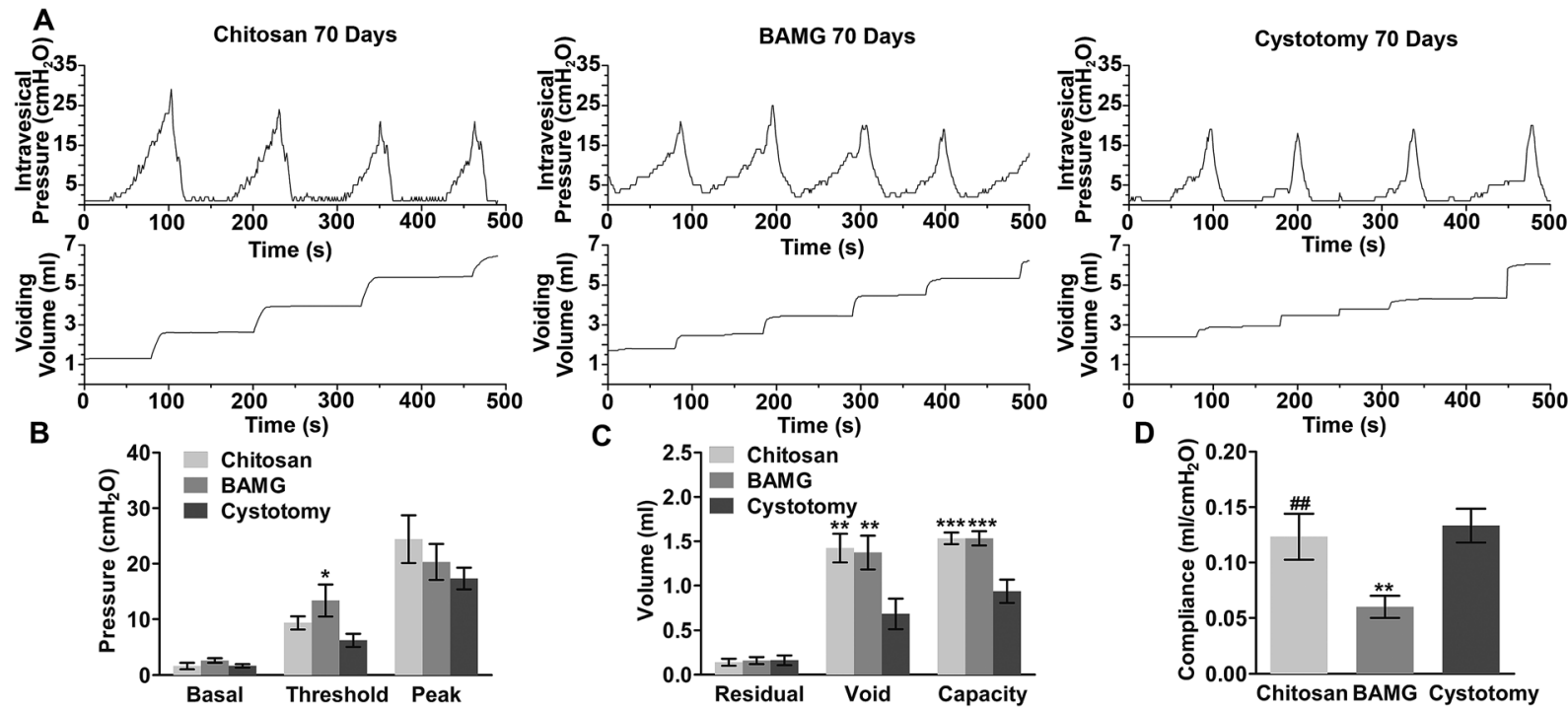

Fig. 6 Cystogram and comparison of urodynamic parameters among the chitosan, BAMG, and cystotomy groups at 70 days after operation. (A) Representative cytograms of the chitosan, BAMG and cystotomy groups. (B) Bladder basal, threshold, and peak pressures at 70 days. (C) Residual, void volumes, and bladder capacity at 70 days. (D) Bladder compliance at 70 days. $* P<0.05, * * P<0.01, * * * P<0.001$ vs. the cystotomy group; ${ }^{\# \#} P<0.01$ vs. the BAMG group.

bladder capacity). There were no significant differences in residual volume among the three groups (Fig. 6C). The compliance of the chitosan group $\left(0.12 \pm 0.020 \mathrm{ml}\right.$ per $\left.\mathrm{cmH}_{2} \mathrm{O}\right)$ recovered to normal levels as in the cystotomy group $(0.13 \pm$ $0.015 \mathrm{ml}$ per $\mathrm{cmH}_{2} \mathrm{O}$ ), and was significantly higher than that of the BAMG group $\left(0.060 \pm 0.010 \mathrm{ml}\right.$ per $\left.\mathrm{cmH}_{2} \mathrm{O}\right)(P<0.01)$ (Fig. 6D). These results suggested that the regenerated bladder using the chitosan scaffold led to appropriate bladder function.

Scaffold porosity has long been regarded to affect angiogenesis and the development of functional tissue in bladder 
reconstruction. ${ }^{38-40}$ The bilayer chitosan scaffold fostered more angiogenesis than BAMG by achieving comparable vessel numbers with control group. It was consistent with a previous study that a porous silk fibroin/chitosan blend scaffold prompted uniform vascularization in hernia repair when compared to relative dense acellular dermal matrix. ${ }^{41}$ Moreover, approximate $10 \%$ increase of porosity promoted smooth muscle regeneration and vessel density in smooth muscle cells-seeded composite scaffold of BAMG and electro-spun PLGA scaffold. ${ }^{39}$ In the present study, the bladder was augmented by the chitosan scaffold with 1.5 -fold capacity with normal compliance. The promoted bladder function is partially attributed to the increased smooth muscle proportion, whose contractile ability was closely linked to bladder function. ${ }^{42}$ In addition, the controllable porosity of chitosan scaffolds was beneficial to angiogenesis, which is fundamental in supporting the survival and function of the regenerated soft tissues. ${ }^{43}$

\section{Chitosan scaffold enhanced angiogenesis through the SDF- 1a/CXCR4 pathway}

Next, we formed a preliminary exploration of the possible effects of the chitosan scaffold on angiogenesis. Therefore, we analyzed the expression of VEGF and VEGFR2. The results showed that the SDF- $1 \alpha$ and CXCR4 mRNA was upregulated in the chitosan group compared to the BAMG group $(P<0.05$ and $P$ $<0.001$, respectively) (Fig. 7A and B, ESI Table 1 and ESI Fig. $3 \dagger$ ), which were consistent with their protein expressions (Fig. 7E). SDF- $1 \alpha$ is an upstream protein of the VEGF, and has many functions, including synergetic angiogenetic effects with VEGF and chemotaxis of endothelial progenitor cells. ${ }^{44,45}$ We found that VEGF and VEGFR2 were significantly increased in the chitosan group compared to the BAMG group (Fig. 7), both at the transcriptional and protein levels, suggesting that the enhanced angiogenetic potential of the chitosan scaffold was associated with the activation of the SDF- $1 \alpha /$ CXCR4 pathway.

The underlying mechanisms of larger porosity resulting in increased regeneration of smooth muscle and angiogenesis are still unclear. One possible explanation is that blood vessel infiltration happens more rapidly as the density of the pores increases. ${ }^{46}$ It was also reported that a mesoporous glass nanolayer increased the gene expression of VEGF and hypoxia inducible factor-1 in human umbilical vein endothelial cells. ${ }^{47}$ Diffusion-limitation is an ever-present problem in tissue engineering, and scaffolds of any reasonable magnitude that fail to account for diffusion will ultimately suffer from areas of hypoxia. On the basis of this study, it was speculated that the activation of the hypoxia-related SDF-1 $\alpha /$ CXCR4 pathway by the bilayer chitosan scaffold could be another important factor contributing to its enhanced angiogenic effects. The hypoxia stimulation caused by the regeneration process in the porous chitosan scaffold activated the SDF-1 $\alpha /$ CXCR 4 pathway by secreting SDF- $1 \alpha$ and expressing CXCR4, regulating the mobilization and chemotaxis of hemangioblasts, secretion of matrix metalloproteinases for enhancing endothelial progenitor cell engraftment, secretion of angiopoietic factors such as VEGF, and reduced apoptosis of endothelial cells. ${ }^{48}$ In the present study, the chitosan scaffold upregulated the gene and protein expression of VEGF and its receptor VEGFR2 in bladders, which then induced hemangioblasts to form angiogenic network structures. The synergistic effects of SDF-1 $\alpha$ and VEGF stimulated the proliferation and tube formation of endothelial cells, which further enhanced angiogenesis. ${ }^{49}$ Nevertheless, the development of blood vessels in tissue engineering is a complicated process involving numerous angiopoietic growth factors. ${ }^{50}$ More efforts should be made to determine the detailed mechanisms of the angiogenic potential of the chitosan scaffold and enhance the maturity of the regenerated blood vessels.
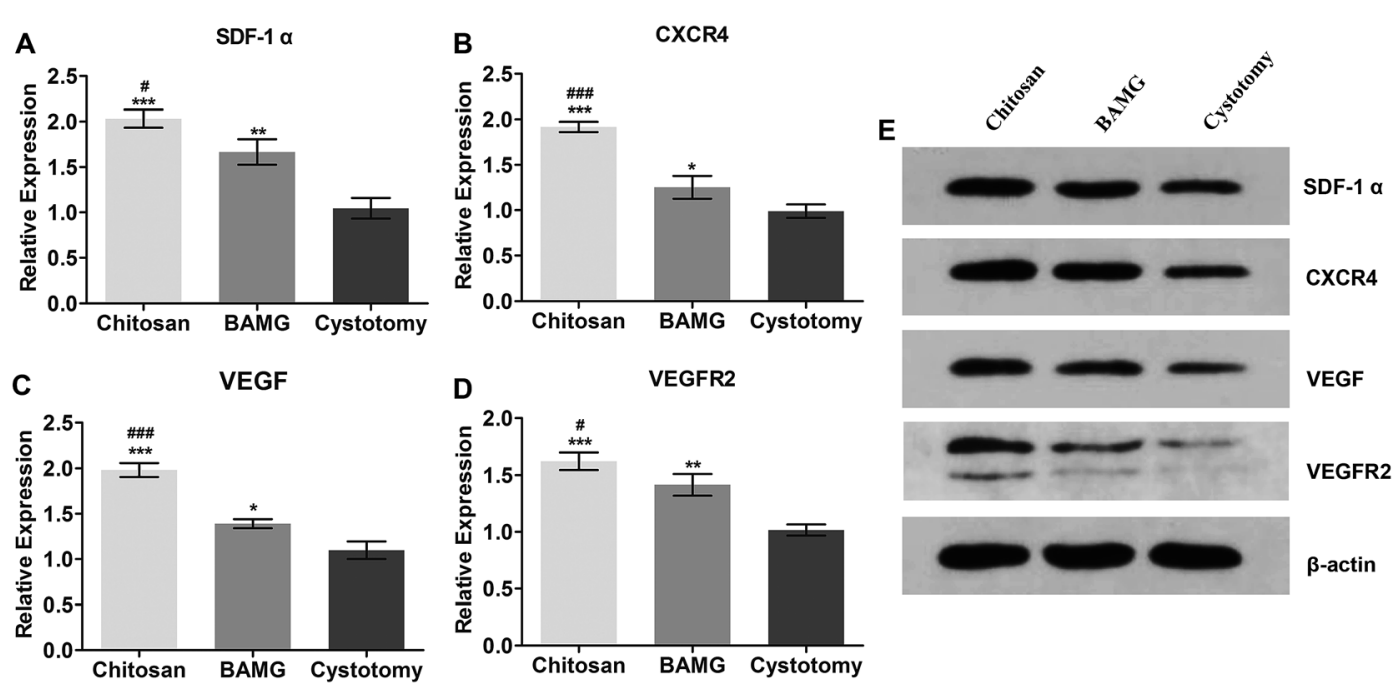

Fig. 7 The chitosan scaffold enhanced angiogenesis by inducing VEGF through the activation of the SDF-1 $\alpha / C X C R 4$ pathway. Expression levels of SDF-1 $1 \alpha(A)$, CXCR4 (B), VEGF (C), and VEGFR2 (D) were evaluated by qRT-PCR using the bladders of the chitosan, BAMG, and cystotomy groups at 70 days post-implantation. (E) Western blot analysis of SDF-1 $\alpha$, CXCR4, VEGF, and VEGFR2 in the bladder of the three groups at 70 days

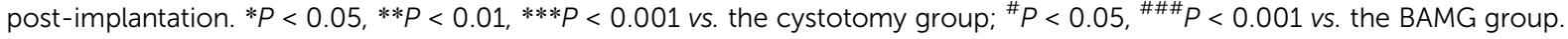




\section{Conclusions}

In general, these are clear advantages of the asymmetric chitosan scaffold applied in our study in comparison with available materials for bladder reconstruction. ${ }^{51}$ The insufficient mechanical strength of collagen biomatrix and the lack of waterproofness of single-layered porous synthetic scaffolds are likely to induce urinary ascites due to scaffold perforation, suture dehiscence or urine leakage, which would result in severe complications. ${ }^{2,38}$ Compared with the traditional flowcasting method, the novel self-deposited chitosan scaffold improves the mechanical properties and does not become curly in its operational environment. ${ }^{22}$ The bilayer design of one compact layer and one porous layer has a combination of proper mechanical strength, elasticity, porosity and water tightness, which are fundamental for the initial bladder defect reinforcements, subsequent regeneration and final functional recovery.

It should be admitted that the bilayer chitosan scaffold needs some modifications. Many different substrates and bioactive molecules have been added into chitin-based scaffold to increase their affinity with nerve cells, such as laminin peptides. ${ }^{52}$ Mesenchymal stem cells, angiogenetic and neurotrophic growth factors could also be applied to further promote angiogenesis and innervation. More mechanical investigations in the regenerated bladder are warranted to examine the mechanical behavior of the incorporated bilayer chitosan, including uniaxial tension test and bladder wall tension. ${ }^{53}$ Additional experiments in large animals are necessary to substantiate the feasibility of the chitosan scaffold for its clinical application.

The present study showed that the bilayer chitosan scaffold had better performances than BAMG in radiographic cystography, smooth muscle regeneration, blood vessel numbers, and bladder function restoration, indicating its promising potential for bladder reconstruction.

\section{Authors' contributions}

D. X., Q. H., A, Q. and M. L. designed this study, X. L. evaluated the scaffold parameters, Q. W. and H. Y. performed the animal experiments, Y. Z. and M. Z. carried out morphological and histological evaluations of regenerated bladder, Z. Z. and J. X. performed the cystometry.

\section{Conflicts of interest}

There are no conflicts to declare.

\section{Acknowledgements}

This study was supported by National Natural Science Foundation of China $(81670622,81370860)$ and the Interdisciplinary Program of Shanghai Jiao Tong University (YG2011MS14, YG2015QN02, YG2016QN47).

\section{References}

1 M. C. Adams and D. B. Joseph, in Walsh-Campbell Urology, ed. A. J. Wein, L. R. Kavoussi, A. C. Novick, A. W. Partin and C. A. Peters, Saunders Elsevier, Philadelphia, 2007.

2 R. D. Cespedes and E. J. McGuire, in Glenn's Urology, ed. J. F. Glenn, S. D. Graham and T. E. Keane, Lippincott Williams and Wilkins, Philadelphia, 2004.

3 D. M. Dahl and W. S. McDougal, in Campbell-Walsh Urology, ed. A. J. Wein, L. R. Kavoussi, A. C. Novick, A. W. Partin and C. A. Peters, Saunders Elsevier, Philadelphia, 2007.

4 T. S. Lendvay, C. A. Cowan, M. M. Mitchell, B. D. Joyner and R. W. Grady, J. Urol., 2006, 176, 1716-1720.

5 P. D. Metcalfe and R. C. Rink, Curr. Urol. Rep., 2007, 8, 152-156.

6 B. Shekarriz, J. Upadhyay, S. Demirbilek, J. S. Barthold and R. Gonzalez, Urology, 2000, 55, 123-128.

7 J. Adamowicz, T. Kowalczyk and T. Drewa, Cent. Eur. J. Urol., 2013, 66, 202-206.

8 W. A. Farhat, J. Urol., 2014, 192, 1021-1022.

9 M. Sloff, V. Simaioforidis, R. de Vries, E. Oosterwijk and W. Feitz, J. Urol., 2014, 192, 1035-1042.

10 A. Atala, S. B. Bauer, S. Soker, J. J. Yoo and A. B. Retik, Lancet, 2006, 367, 1241-1246.

11 D. B. Joseph, J. G. Borer, R. E. De Filippo, S. J. Hodges and G. A. McLorie, J. Urol., 2014, 191, 1389-1395.

12 P. Caione, R. Boldrini, A. Salerno and S. G. Nappo, Pediatr. Surg. Int., 2012, 28, 421-428.

13 J. P. Zambon, L. S. de Sa Barretto, A. N. Nakamura, S. Duailibi, K. Leite, R. S. Magalhaes, G. Orlando, C. L. Ross, A. Peloso and F. G. Almeida, Organogenesis, 2014, 10, 278-288.

14 L. Song, S. V. Murphy, B. Yang, Y. Xu, Y. Zhang and A. Atala, Tissue Eng., Part B, 2014, 20, 163-172.

15 Z. Zhou, M. Zhang, M. Xu, K. Zhang, Y. Zhao, J. Zhou, Y. Zhu, Z. Wang and M. Lu, Biotechnol. Bioprocess Eng., 2015, 20, 523-531.

16 Y. Zhang, D. Frimberger, E. Y. Cheng, H. K. Lin and B. P. Kropp, BJU Int., 2006, 98, 1100-1105.

17 W. D. Zhu, Y. M. Xu, C. Feng, Q. Fu and L. J. Song, Urologe A., 2011, 50, 1420-1425.

18 C. Shi, Y. Zhu, X. Ran, M. Wang, Y. Su and T. Cheng, J. Surg. Res., 2006, 133, 185-192.

19 T. Freier, H. S. Koh, K. Kazazian and M. S. Shoichet, Biomaterials, 2005, 26, 5872-5878.

20 S. G. Hu, C. H. Jou and M. C. Yang, Biomaterials, 2003, 24, 2685-2693.

21 S. V. Madihally and H. W. Matthew, Biomaterials, 1999, 20, 1133-1142.

22 L. Yang, W. Lu, Y. Pang, X. Huang, Z. Wang, A. Qin and Q. $\mathrm{Hu}, R S C A d v .$, 2016, 6, 71567-71573.

23 Y. Zhao, Y. He, Z. Zhou, J. H. Guo, J. S. Wu, M. Zhang, W. Li, J. Zhou, D. D. Xiao, Z. Wang, K. Sun, Y. J. Zhu and M. J. Lu, Acta Biomater., 2015, 23, 91-102.

24 L. Xu, D. Cao, W. Liu, G. Zhou, W. J. Zhang and Y. Cao, Biomaterials, 2010, 31, 3894-3902. 
25 Z. Song, Z. Peng, Z. Liu, J. Yang, R. Tang and Y. Gu, Tissue Eng., Part A, 2013, 19, 1543-1553.

26 D. D. Tu, A. Seth, E. S. Gil, D. L. Kaplan, J. R. Mauney and C. R. Estrada Jr, J. Visualized Exp., 2012, 2012(66), e3981.

27 A. K. Sharma, M. I. Bury, N. J. Fuller, A. J. Marks, D. M. Kollhoff, M. V. Rao, P. V. Hota, D. J. Matoka, S. L. Edassery, H. Thaker, J. F. Sarwark, J. A. Janicki, G. A. Ameer and E. Y. Cheng, Proc. Natl. Acad. Sci. U. S. A., 2013, 110, 4003-4008.

28 H. K. Lin, S. V. Madihally, B. Palmer, D. Frimberger, K. M. Fung and B. P. Kropp, Adv. Drug Delivery Rev., 2015, 82-83, 47-63.

29 J. Prywer and M. Olszynski, Curr. Med. Chem., 2017, 24, 292311.

30 F. Zhang and L. Liao, J. Urol., 2014, 192, 544-550.

31 E. Zakhem, S. Raghavan, R. R. Gilmont and K. N. Bitar, Biomaterials, 2012, 33, 4810-4817.

32 M. Probst, H. J. Piechota, R. Dahiya and E. A. Tanagho, BJU Int., 2000, 85, 362-371.

33 J. Smolar, S. Salemi, M. Horst, T. Sulser and D. Eberli, Transfusion medicine and hemotherapy : offizielles Organ der Deutschen Gesellschaft fur Transfusionsmedizin und Immunhamatologie, 2016, vol. 43, pp. 328-335.

34 J. M. Soria, C. Martinez Ramos, M. Salmeron Sanchez, V. Benavent, A. Campillo Fernandez, J. L. Gomez Ribelles, J. M. Garcia Verdugo, M. M. Pradas and J. A. Barcia, J. Biomed. Mater. Res., Part A, 2006, 79, 495-502.

35 Y. Yuan, P. Zhang, Y. Yang, X. Wang and X. Gu, Biomaterials, 2004, 25, 4273-4278.

36 M. J. Simoes, A. Gartner, Y. Shirosaki, R. M. Gil da Costa, P. P. Cortez, F. Gartner, J. D. Santos, M. A. Lopes, S. Geuna, A. S. Varejao and A. C. Mauricio, Acta Med. Port., 2011, 24, 43-52.

37 B. S. Kim, C. E. Baez and A. Atala, World J. Urol., 2000, 18, 29.

38 D. D. Tu, Y. G. Chung, E. S. Gil, A. Seth, D. Franck, V. Cristofaro, M. P. Sullivan, D. Di Vizio, P. Gomez III, R. M. Adam, D. L. Kaplan, C. R. Estrada Jr and J. R. Mauney, Biomaterials, 2013, 34, 8681-8689.
39 M. Horst, V. Milleret, S. Notzli, S. Madduri, T. Sulser, R. Gobet and D. Eberli, J. Biomed. Mater. Res., Part A, 2014, 102, 2116-2124.

40 A. Seth, Y. G. Chung, E. S. Gil, D. Tu, D. Franck, D. Di Vizio, R. M. Adam, D. L. Kaplan, C. R. Estrada Jr and J. R. Mauney, Biomaterials, 2013, 34, 4758-4765.

41 A. S. Gobin, C. E. Butler and A. B. Mathur, Tissue Eng., 2006, 12, 3383-3394.

42 C. H. Fry, E. Meng and J. S. Young, Autonomic neuroscience : basic \& clinical, 2010, vol. 154, pp. 3-13.

43 Y. G. Ko, N. Kawazoe, T. Tateishi and G. Chen, J. Biomed. Mater. Res., Part B, 2010, 93, 341-350.

44 J. M. Tang, J. N. Wang, L. Zhang, F. Zheng, J. Y. Yang, X. Kong, L. Y. Guo, L. Chen, Y. Z. Huang, Y. Wan and S. Y. Chen, Cardiovasc. Res., 2011, 91, 402-411.

45 J. Kijowski, M. Baj-Krzyworzeka, M. Majka, R. Reca, L. A. Marquez, M. Christofidou-Solomidou, A. JanowskaWieczorek and M. Z. Ratajczak, Stem cells, 2001, 19, 453-466. 46 C. M. Walthers, A. K. Nazemi, S. L. Patel, B. M. Wu and J. C. Dunn, Biomaterials, 2014, 35, 5129-5137.

47 Y. Zhang, L. Xia, D. Zhai, M. Shi, Y. Luo, C. Feng, B. Fang, J. Yin, J. Chang and C. Wu, Nanoscale, 2015, 7, 19207-19221.

48 Y. Wang and K. Luther, Prog. Mol. Biol. Transl. Sci., 2012, 111, 265-284.

49 M. Kucia, K. Jankowski, R. Reca, M. Wysoczynski, L. Bandura, D. J. Allendorf, J. Zhang, J. Ratajczak and M. Z. Ratajczak, J. Mol. Histol., 2004, 35, 233-245.

50 E. C. Novosel, C. Kleinhans and P. J. Kluger, Adv. Drug Delivery Rev., 2011, 63, 300-311.

51 M. Rodriguez-Vazquez, B. Vega-Ruiz, R. Ramos-Zuniga, D. A. Saldana-Koppel and L. F. Quinones-Olvera, BioMed Res. Int., 2015, 2015, 821279.

52 A. Matsuda, H. Kobayashi, S. Itoh, K. Kataoka and J. Tanaka, Biomaterials, 2005, 26, 2273-2279.

53 F. G. Habteyes, S. O. Komari, A. S. Nagle, A. P. Klausner, R. L. Heise, P. H. Ratz and J. E. Speich, J. Mech. Behav. Biomed. Mater., 2017, 71, 192-200. 